\title{
Activation of AMPK inhibits cervical cancer cell growth through AKT/FOXO3a/FOXM1 signaling cascade
}

\author{
Mingo Ming Ho Yung ${ }^{1}$, David Wai Chan ${ }^{1 *}$, Vincent Wing Sun Liu' ${ }^{1}$ Kwok-Ming Yao ${ }^{2}$ \\ and Hextan Yuen-Sheung Ngan ${ }^{1^{*}}$
}

\begin{abstract}
Background: Although advanced-stage cervical cancer can benefit from current treatments, approximately 30\% patients may fail after definitive treatment eventually. Therefore, exploring alternative molecular therapeutic approaches is imperatively needed for this disease. We have recently shown that activation of AMP-activated protein kinase (AMPK), a metabolic sensor, hampers cervical cancer cell growth through blocking the Wnt/ $\beta$-catenin signaling activity. Here, we report that activated AMPK ( $p-A M P K$ ) also inhibits cervical cancer cell growth by counteracting FOXM1 function.

Methods: Effect of the activation of AMPK on FOXM1 expression was examined by hypoxia and glucose deprivation, as well as pharmacological AMPK activators such as A23187, AICAR and metformin. RT Q-PCR and Western blot analysis were employed to investigate the activities of AMPK, FOXM1 and AKT/FOXO3a signaling.

Results: Consistent with our previous findings, the activation of AMPK by either AMPK activators such as AICAR, A23187, metformin, glucose deprivation or hypoxia significantly inhibited the cervical cancer cell growth. Importantly, we found that activated AMPK activity was concomitantly associated with the reduction of both the mRNA and protein levels of FOXM1. Mechanistically, we showed that activated AMPK was able to reduce AKT mediated phosphorylation of p-FOXO3a (Ser253). Interestingly, activated AMPK could not cause any significant changes in FOXM1 in cervical cancer cells in which endogenous FOXO3a levels were knocked down using siRNAs, suggesting that FOXO3a is involved in the suppression of FOXM1.
\end{abstract}

Conclusion: Taken together, our results suggest the activated AMPK impedes cervical cancer cell growth through reducing the expression of FOXM1.

Keywords: AMPK, FOXM1, FOXO3a, Cervical cancer

\section{Background}

Cervical cancer results from uncontrolled growth of malignant cells started within the uterine cervix and is one of the most common malignancies in women worldwide [1-3]. Although this disease is almost preventable with routine genetic screening and vaccination, more than $80 \%$ of cervical cancers with a majority in the advanced stage are currently found in developing countries including China, leading to a high risk of recurrence and poor

\footnotetext{
* Correspondence: dwchan@hku.hk; hysngan@ hku.hk

${ }^{1}$ Departments of Obstetrics and Gynaecology, LKS Faculty of Medicine, The University of Hong Kong, Hong Kong SAR, People's Republic of China Full list of author information is available at the end of the article
}

survival $[2,4]$. Thus, there is a compelling need to explore novel therapeutic interventions for this disease.

Emerging evidence suggests that targeting cancer cell metabolism is a promising therapeutic approach in human cancers. AMP-activated protein kinase (AMPK) is a known cellular metabolic sensor and plays an important role in the control of energy homeostasis in response to external stresses [5-8]. Recent studies have documented that pharmacological activation of AMPK is able to block cancer cell growth in various human cancers [8-11]. Indeed, we have previously reported that pharmaceutical AMPK activators such as AICAR (ATP-dependent) and A23187 (ATP-independent) could suppress cervical cancer cell

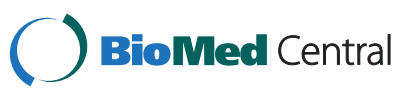


growth in the presence or absence of LKB1, an upstream kinase of AMPK [10]. We also proposed mechanistic evidence showing that metformin, AICAR and A23187 suppress cervical cancer cell growth through reducing DVL3, a positive effector of $\mathrm{Wnt} / \beta$-catenin signaling cascade which has been shown to be constitutively active during cervical cancer development [12]. Yet, it is still believed that there are other molecular mechanisms by which these pharmaceutical AMPK activators suppress cancer cell growth. The understanding of these mechanisms will assist in exploring better therapeutic regimes when using these drugs.

Forkhead Box M1 (FOXM1) is a member of the Forkhead Box transcription factors which is essential for cell proliferation and apoptosis in the development and function of many organs [13-17]. We previously reported that aberrant upregulation of FOXM1 is associated with the progression and development of human cervical squamous cell carcinoma (SCC) [18]. Biochemical and functional studies confirmed that FOXM1 is critically involved in cervical cancer cell growth through upregulating cyclin B1, cyclin D1 and cdc25B and downregulating p27 and p21 expressions. These findings suggest that FOXM1 plays a vital role in cervical cancer cell growth and oncogenesis.

In this study, we reported that the activated AMPK inhibits the cell growth by reducing FOXM1 expression in human cervical cancer cells upon treatments with hypoxia, glucose deprivation and pharmaceutical AMPK activators. We provided both biochemical and functional evidence to support our findings that the repression of FOXM1 expression of AMPK is dependent on the AKT/FOXO3a/FOXM1 signaling cascade.

\section{Methods}

\section{Cell lines and reagents}

Cervical cancer cell lines HeLa, CaSki, C33A and SiHa (American Type Culture Collection, Rockville, Md., USA) (cell line authentication was done by in-house STR DNA profiling analysis) were employed in this study. They were maintained in Dulbecco's Modified Eagle Medium (DMEM) (Invitrogen, Carlsbad, CA) supplemented with $10 \%(\mathrm{v} / \mathrm{v})$ fetal bovine serum (Gibco), 100 units $/ \mathrm{ml}$ penicillin/streptomycin (Gibco) at $37^{\circ} \mathrm{C}$ in an incubator with humidified atmosphere of $5 \% \mathrm{CO}_{2}$ and 95\% air. AMPK activators AICAR, A23187 and metformin and AKT inhibitor LY294002 were obtained from Tocris Bioscience (Bristol, UK). FOXM1 inhibitor Thiostrepton was purchased from Calbiochem (La Jolla, CA, USA).

\section{Plasmids and cell transfection}

To study the effects of enforced FOXM1 expression, the FOXM1c-expressing plasmid pcDNA3-FOXM1c was used because the $\mathrm{c}$ isoform has higher transactivating activity and is expressed dominantly in cells as well as tissues. Whereas, the pcDNA3 empty vector was used in mock transfections as control. Besides, the vector-based shRNA plasmid pTER-FOXM1 was used to knockdown endogenous FOXM1. All of these plasmids had been described previously [18]. As controls in knockdown assays, the p-super GFP and pcDNA3 vectors were used in mock transactions. To knockdown human FOXO3a, the TriFECTa RNAi Kit which contains three siRNAs targeting human FOXO3a was purchased from IDT (Integrated DNA Technologies, Inc., Iowa, USA). Cell transfection was carried out using LipofectAMINETM 2000 (Invitrogen) according to the manufacturer's instructions. Expression patterns were analyzed by Western blotting. The parental vector pEGFP-C1 was used as empty vector control.

\section{Cell proliferation assay}

Cell proliferation kit (XTT) (Roche, Basel, Switzerland) was used to measure cell viability according to the manufacturer's protocol. Three independent experiments were performed in triplicates.

\section{RNA extraction and quantitative reverse transcriptase- PCR (Q-PCR)}

According to the instruction of the manufacturer, total RNA was extracted using TRIzol reagent (Invitrogen). Complementary DNA (cDNA) was subsequently synthesized using a reverse transcription reagent kit (Applied Biosystems, Foster City). The expression level of FOXM1 was then evaluated by q-PCR in an ABI PRISM ${ }^{\text {tw }} 7500$ system (Applied Biosystems) using Taqman ${ }^{\circ}$ Gene expression Assays; human FOXM1 (Assay ID: Hs00153543_m1). The human 18S rRNA (Assay ID: Hs99999901_m1) was used as an internal control.

\section{Western blot analysis}

Proteins in cell lysates were separated by 10\% SDS-PAGE and transferred to polyvinylidene-difluoride (PVDF) membranes. The membranes were blotted with $5 \%$ skimmed milk and subsequently probed overnight at $4^{\circ} \mathrm{C}$ with primary antibodies specific for p-AMPK $\alpha$, AMPK $\alpha, \mathrm{p}$-AKT, AKT, p-FOXO3a, FOXO3a (Cell Signaling, Beverly, MA, USA), FOXM1 (Santa Cruz Biotechnology, Inc., Santa Cruz, CA, USA) and $\beta$-actin (Sigma-Aldrich, St. Louis, MO, USA) and then incubated with horseradish peroxidase conjugated goat anti-rabbit or anti-mouse secondary antibody (Amersham, Uppsala, Sweden). Immunodetection was performed with enhanced chemiluminescent reagent solution (Amersham ${ }^{\mathrm{TM}} \mathrm{ECL}^{\mathrm{TM}}$ ) and visualized using medical X-ray film. 


\section{Data analysis}

Student's $t$ test was applied to the data analysis. All data were expressed as mean \pm SEM. P-value of less than 0.05 was considered as significant.

\section{Results}

Increased AMPK activity inhibits cervical cancer cell growth by suppressing FOXM1 expression

Consistent with our previous findings [12], this study also showed that the cell growth of cervical cancer cell lines such as Caski and $\mathrm{SiHa}$ was significantly inhibited by the AMPK activator metformin on a time and dose dependent manner (Figure 1A). Similarly, other AMPK activators such as AICAR and A23187 displayed remarkable inhibitory effect on cervical cancer cells (data not shown), confirming that activation of AMPK is able to reduce cervical cancer cell growth.
As FOXM1 is a master regulator of cancer cell growth, it is of interest to examine whether increased AMPK activity has any functional impact on FOXM1 in cervical cancer oncogenesis. Upon treatment of AICAR (1 mM), A23187 $(2 \mu \mathrm{M})$, metformin $(25 \mathrm{mM})$, glucose deprivation and hypoxia on the cervical cancer cell lines Caski, C33A and HeLa, we found that FOXM1 expression was drastically decreased while AMPK activity [p-AMPK (Thr172)] was elevated concomitantly (Figure 1B and Figure 2). Interestingly, Q-PCR analysis also demonstrated that the mRNA level of FOXM1 in C33A and $\mathrm{SiHa}$ cells was remarkably reduced upon treatment of metformin $(25 \mathrm{mM})$ and A23187 $(2 \mu \mathrm{M})$ in a time dependent manner (Figure $1 \mathrm{C}$ ). This finding indicates that AMPK activated by these pharmaceutical activators could suppress FOXM1 expression at both protein and mRNA levels.
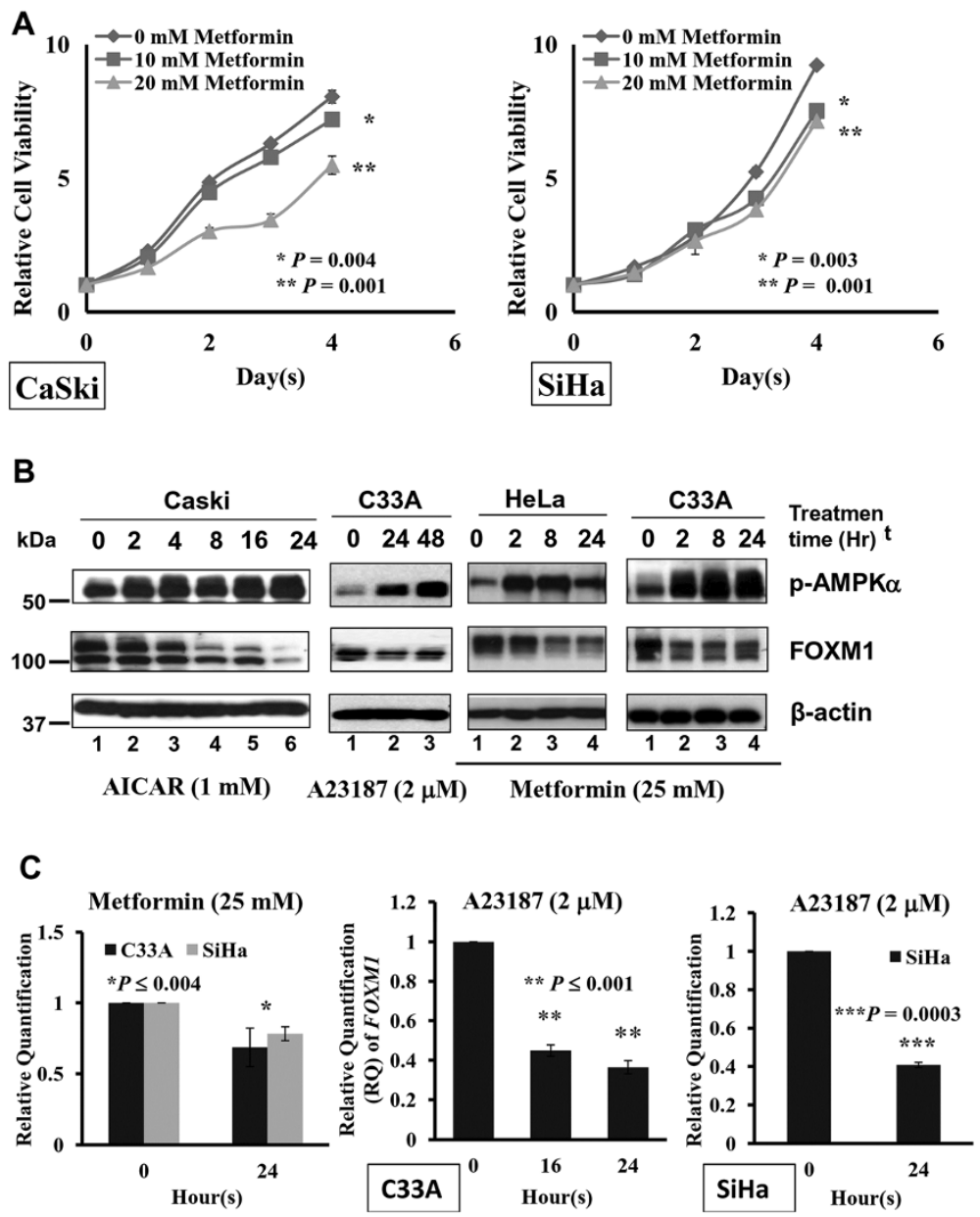

Figure 1 Activation of AMPK by pharmaceutical AMPK activators suppresses expression of FOXM1. (A) XTT cell proliferation assay showed that the growth rates of cervical cells; Caski and SiHa, were significantly reduced in a dose and time dependent manner upon the treatment of metformin (10 mM and $20 \mathrm{mM}$ ) when compared with the control medium. (B) Western blot analysis showed that the level of p-AMPKa was increased, while the level of FOXM1 was reduced in cervical cancer cells (Caski, C33A and Hela) when treated with AICAR (1 mM), A23187 (2 $\mu$ M) and metformin (25 mM) for various time points. (C) RT-Q-PCR analysis demonstrated that the FOXM1 mRNA level of C33A and SiHa cells was remarkably reduced when treated with metformin $(25 \mathrm{mM})$ and A23187 $(2 \mu \mathrm{M})$ from 16 hrs to 24 hrs. The 185 gene was used as an internal control. 


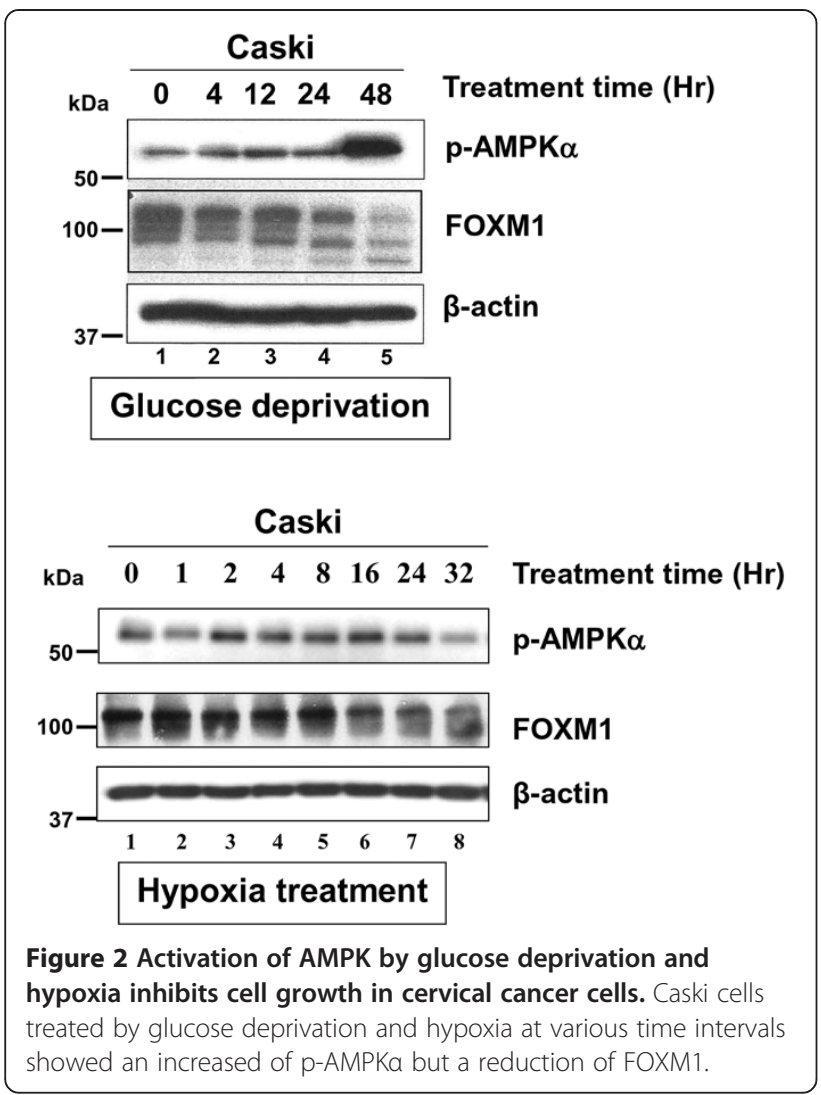

Activated AMPK represses FOXM1 expression through blocking the AKT/FOXO3a signaling pathway

FOXO3a is well known to be one of the FOXO transcription factors that functions downstream of PI3K-PTEN-AKT (PKB) signaling in modulating cell growth [19]. Along the signaling cascade, FOXO3a is a negative regulator of FOXM1 expression [20]. To investigate whether the suppressive effect of AMPK on FOXM1 is mediated via FOXO3a, we first examined the intensity of FOXO3a dephosphorylation by metformin. Upon treatment of metformin $(25 \mathrm{mM})$ on the cervical cancer cell line C33A, not only FOXM1 expression diminished remarkably but also the phosphorylation of AKT and the AKT-specific phosphorylation of FOXO3a (Ser253) (Figure 3A). We then examined whether the PI3K/AKT inhibitor LY294002 could reduce FOXM1 expression in cervical cancer cells. As expected, C33A cells exhibited decreased intensity of AKT-specific phosphorylation of FOXO3a (Ser253) as well as FOXM1 expression upon the treatment of LY294002 (10 $\mu \mathrm{M})$ (Figure 3B). To further assess whether FOXO3a is primarily involved in the reduction of FOXM1 induced by AMPK activation but not an off-target pharmaceutical effect, siRNA-based knockdown of FOXO3a in C33A cells was carried out. Western blot analysis revealed that cervical cancer cells with depletion of endogenous FOXO3a did not show altered FOXM1

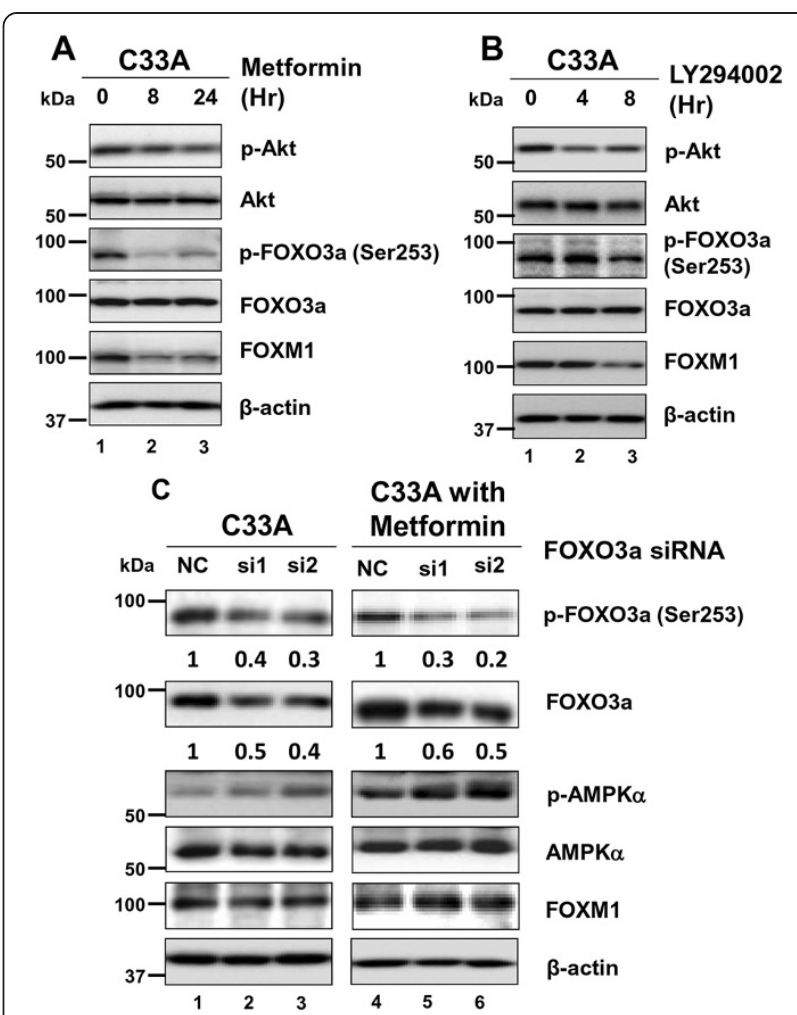

Figure 3 Activation of AMPK represses FOXM1 expression through inhibiting the AKT/FOXO3a signaling pathway. (A) Upon treatment of metformin (25 mM) in cervical cancer cells (C33A), the expression of FOXM1 diminished remarkably and the phosphorylation of AKT and AKT-specific phosphorylation of FOXO3a (Ser253) decreased concomitantly. (B) Co-treatment of PI3K AKT inhibitor, LY294002 (10 HM), inhibited p-AKT and concomitantly reduced the levels of FOXO3a (Ser253) and FOXM1 in C33A cells. (C) Depletion of endogenous FOXO3a in C33A cells using siRNAs showed that FOXO3a expression is required for the suppression of FOXM1 by metformin $(25 \mathrm{mM})$ treatment.

expression even when AMPK was activated subsequently by metformin (25 mM) (Figure 3C). Taken together, our data support that inhibition of FOXM1 by AMPK activation is attributed to the repression on AKT and its downstream, AKT-specific phosphorylation of FOXO3a (Ser253) in cervical cancer cells.

Ectopic expression of FOXM1 rescues AMPK-mediated cell growth inhibition

Given that activation of AMPK leads to growth inhibition of cervical cancer cells through reduction of both the mRNA and protein levels of FOXM1, we sought to determine whether enforced expression of exogenous FOXM1 could counteract the AMPK-induced suppressive effect. C33A and SiHa cells were transiently transfected with FOXM1c-expressing plasmid and treated with metformin (20 mM). Consistent with our previous findings [18], XTT cell proliferation analysis showed that enforced expression 
of FOXM1c significantly promoted cell proliferation in both C33A $(P=0.00007)$ and $\mathrm{SiHa}(P=0.0004)$ cells as compared with the vector control (Figure 4A). Importantly, C33A and SiHa cells with ectopic expression of FOXM1c could significantly reduce the effect of AMPK-mediated cell proliferation inhibition as compared with their vector controls upon treatment of metformin (20 mM) (Figure 4A). Such counteracting effect of ectopic FOXM1c was particularly evident in $\mathrm{SiHa}$ cells (Figure 4A). Indeed, Western blot analysis confirmed that there was no reduction in the expression of FOXM1 in FOXM1c-transfected $\mathrm{SiHa}$ cells upon treatment of metformin $(25 \mathrm{mM})$ for $24 \mathrm{hrs}$ (Figure 4B). Collectively, these findings confirm that activation of AMPK by hypoxia and glucose deprivation, as well as pharmacological AMPK activators inhibits cervical cancer cell growth, and this effect is dependent on the endogenous expression level of FOXM1.

\section{FOXM1 acts as an AMPK downstream effector}

Previous experiments have demonstrated that reduction of FOXM1 is a common scenario when AMPK is activated in cervical cancer cells. As FOXM1 is a key transcription factor, we sought to determine whether alteration of FOXM1 levels causes a feedback control on AMPK activity. To this end, we treated C33A cells with the FOXM1 specific inhibitor thiostrepton to suppress FOXM1 expression. Upon treatment of thiostrepton $(5 \mu \mathrm{M})$, FOXM1 expression was significantly suppressed, whereas the expression of p-AMPK $\alpha$ (Thr172) was unchanged (Figure 4C). Similarly, knockdown of endogenous FOXM1 using shRNAs did not reveal any discernible change on the expression level of p-AMPK $\alpha$ (Thr172) in C33A cells (Figure 4D). Taken together, p-AMPK $\alpha$ activity per se is not altered by FOXM1 suppression induced by thiostrepton treatment or shRNA knockdown, implying

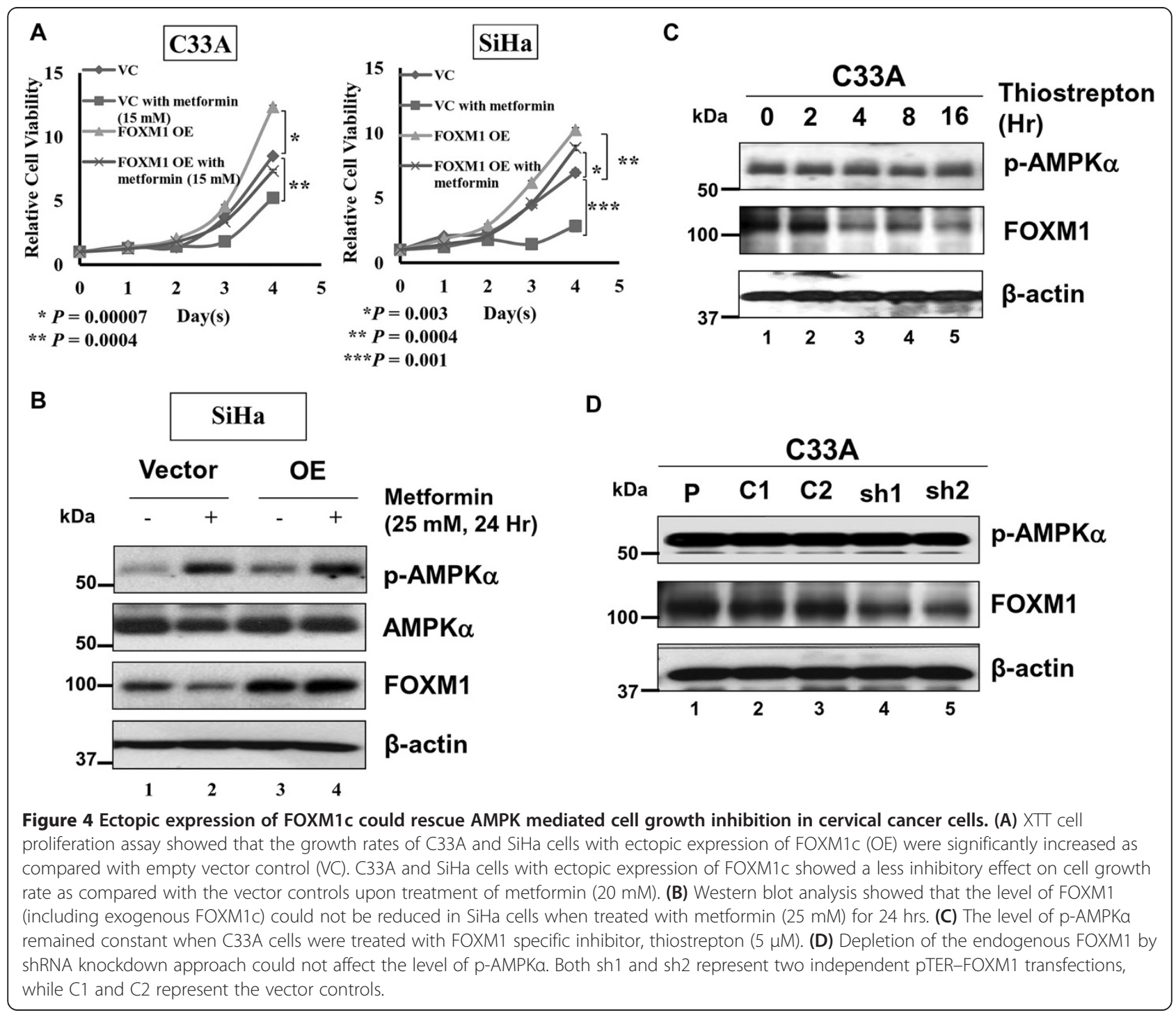


that AMPK is acting upstream of FOXM1 and there is no feedback loop. Our analysis strongly supports that increased AMPK activity down-regulates FOXM1 through the AKT/FOXO3a/FOXM1 signaling cascade (Figure 5).

\section{Discussion}

Recent studies have suggested that targeting cancer cell metabolism is an alternative therapeutic approach in cancer treatment. AMPK is a pivotal energy sensor governing normal and cancer cell metabolism. Our previous research has shown that pharmaceutical AMPK activators are able to repress cervical cancer cell growth through targeting DVL3 in the Wnt/ $\beta$-catenin signaling pathway [12]. In this study, we report another molecular mechanism by which AMPK can retard cervical cancer cell growth: inhibition of FOXM1 function via AMPK/AKT/ FOXO3a signaling. We demonstrated that AMPK activated by either micro-environmental stresses or pharmaceutical AMPK activators could reduce FOXM1 expression through blocking the AKT/FOXO3a signaling pathway, that in turn impaired cervical cancer cell growth.

The Forkhead box transcription factor FOXM1 regulates a number of key cell cycle regulators that control the $G_{1}$ to $S$ and the $G_{2}$ to $M$ transitions [21-26]. Accumulating evidences have shown that the upregulation of FOXM1 is often involved in the development of various human cancers [27-32]. We previously reported that there is a progressive increase in FOXM1 level in the progression of human cervical cancer [18]. The inhibition of FOXM1 by genetic or pharmaceutical approach significantly impairs tumor growth of this cancer in vitro and in vivo $[18,25,27,33]$, suggesting that targeting FOXM1 is an appealing and potential approach for anticancer therapeutics. On the other hand, we and others have proved that activation of AMPK is able to inhibit the growth of various human cancers including cervical cancer [10,34-36]. The pharmacological activation of AMPK using AICAR or metformin has been shown to inhibit cell growth and induce cell apoptosis of a wide spectrum of cancer cells through modulation of p53 [37], p27 [38,39], or p21 $[18,40]$, or DVL3 in Wnt//-catenin signaling in cervical cancer [12]. Herein, we demonstrated that the activation of AMPK by various AMPK activators or hypoxia and glucose deprivation stresses induces a remarkable reduction of FOXM1 which in turn leads to a remarkable decrease of cervical cancer cell growth in both HPV positive (Caski, Hela and $\mathrm{SiHa}$ ) and HPV negative (C33A) cell lines. On the other hand, ectopic expression of FOXM1c could counteract the suppressive effect of activated AMPK. These findings indicate that FOXM1 is a key oncogenic factor associated with cervical cancer cell growth, while activated AMPK inhibits cervical cancer cell growth through downregulation of endogenous FOXM1.

In fact, we demonstrated that reduction of FOXM1 occurred at both the mRNA but and protein levels in cervical cancer cells. This is suggestive of a transcriptional suppression of FOXM1 by its upstream effectors. Previous studies have reported that FOXM1 is transcriptionally suppressed by FOXO3a, which is a critical downstream effector of the PI3K/AKT/FOXO signaling pathway $[19,20]$.

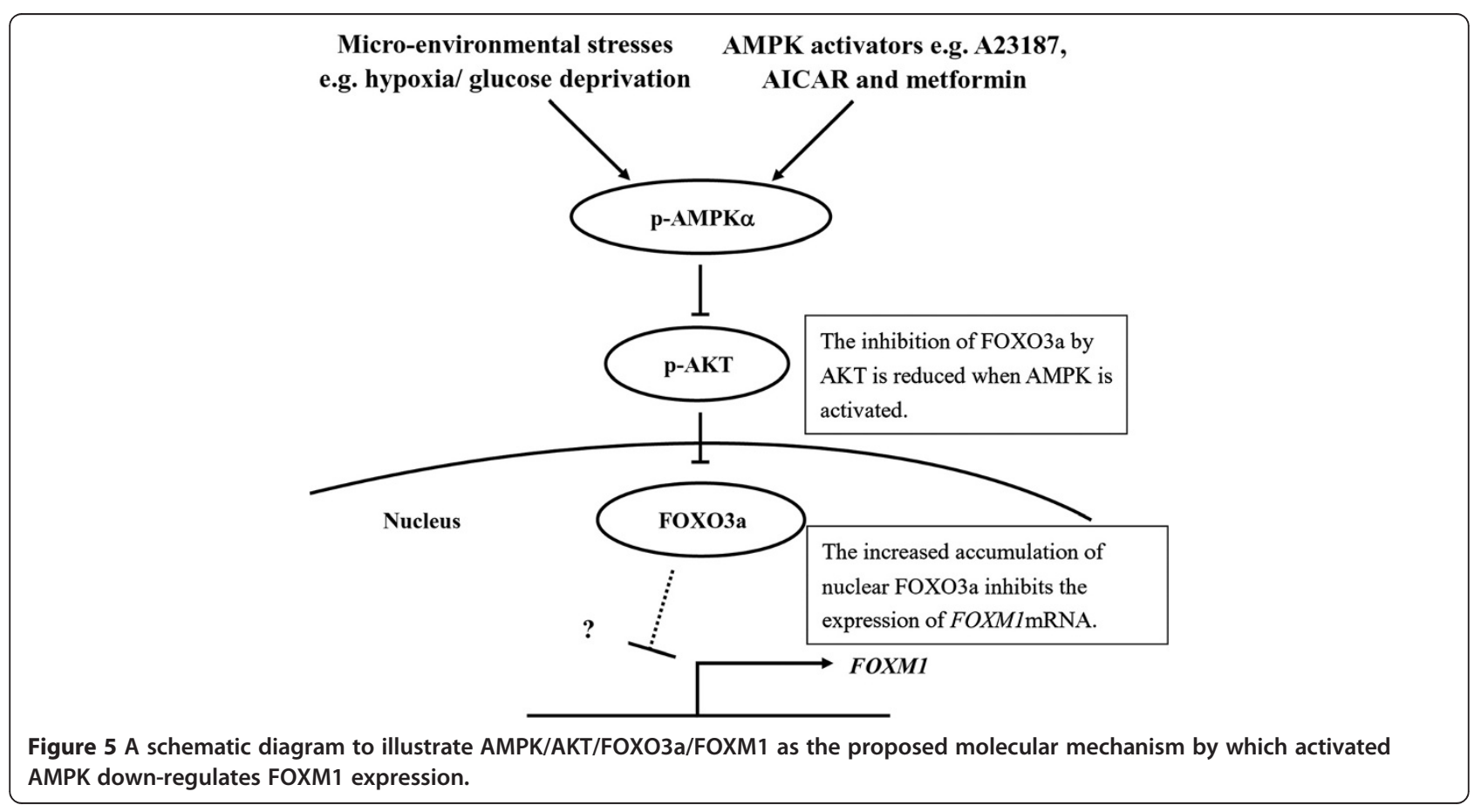


For example, it has been reported that FOXO3a represses estrogen receptors $\alpha(E R \alpha)$ activity in breast cancer cells through an alternative mechanism by which FOXO3a interacts and downregulates the expression of FOXM1 [29,41]. These evidences imply that the reduction of FOXM1 at both the mRNA and protein levels is due to the presence of FOXO3a in cervical cancer cells. In fact, our data using siRNA-mediated FOXO3a knockdown showed that FOXO3a expression is required for FOXM1 reduction upon activation of AMPK.

How do activated AMPK leads to FOXO3a accumulating in the nucleus and blocking the transcription of FOXM1 mRNA? FOXO3a, which belongs to the class O of Forkhead/winged helix box (FOXO) transcription factors, is a key tumor suppressor involved in different cellular processes [42]. FOXO3a is modified by phosphorylation, acetylation and ubiquitination, which in turn affect its nuclear/cytoplasm shuttling, transcriptional activity and stability [43-46]. It is known that the PI3K/AKT signaling is the main regulatory pathway of FOXO3a $[44,47,48]$. When PI3K/AKT signaling is activated, FOXO3a is not only inactivated and phosphorylated at Thr32, Ser253 and Ser315 residues, but is also exported out from the nucleus to the cytoplasm where it is ubiquitinated and subjected to proteasome-dependent degradation $[43,48]$. Therefore, nuclear FOXO3a functions as transcriptional regulator, whereas cytoplasmic FOXO3a is considered inactive [46]. On the other hand, AKT is a signaling kinase known to be inactivated by activated AMPK $[49,50]$. In our study, treatment of either AMPK activator (metformin) or PI3K/AKT inhibitor (LY294002) showed significant inhibition of $\mathrm{p}-\mathrm{AKT}$ and a remarkable reduction of p-FOXO3a (Ser253), an AKT-specific phosphorylation site, suggesting that suppression of FOXO3a is reduced. As a result, FOXO3a will be more nuclear-localized and activated to inhibit FOXM1 mRNA expression in cervical cancer cells.

Aforementioned, AMPK activation can commonly inhibit FOXM1 expression in cervical cancer cells. However, whether there exists a feedback loop on the activity of AMPK is still unknown. To test this notion, cervical cancer cells were treated with the FOXM1 inhibitor thiostrepton to investigate the effect on AMPK activation. Results showed that treatment of thiostrepton only reduced expression of FOXM1 but not the activity of AMPK. In addition, depletion of endogenous FOXM1 using shRNAs gave similar findings as the treatment of thiostrepton, implying that FOXM1 is acting downstream of AMPK without any feedback regulation.

\section{Conclusion}

In summary, our findings here prove that activation of AMPK frequently inhibits cervical cancer cells. More importantly, we demonstrated that activated
AMPK reduces FOXM1 by counteracting the AKT/ FOXO3a/FOXM1 signaling axis. Our findings shed light on the application of AMPK activators in the treatment of human cervical cancer.

\section{Competing interests}

The authors declare that they have no competing interests.

\section{Authors' contributions}

MY and DC designed research; MY, DC and VL performed the experiments; $D C, V L$ and $K Y$ contributed new reagents-analytic tools; MY, DC and HN analyzed and interpreted data; MY and DC wrote the manuscript. All authors were involved in editing the manuscript and had final approval of the submitted and published versions.

\section{Acknowledgements}

This study was supported by Wong Check She Charitable Foundation.

\section{Author details}

${ }^{1}$ Departments of Obstetrics and Gynaecology, LKS Faculty of Medicine, The University of Hong Kong, Hong Kong SAR, People's Republic of China. 2Departments of Biochemistry, LKS Faculty of Medicine, The University of Hong Kong, Hong Kong SAR, People's Republic of China.

Received: 8 April 2013 Accepted: 1 July 2013

Published: 3 July 2013

\section{References}

1. Yang HJ, Liu WW, Tsang PC, Yip AM, Tam KF, Wong LC, Ng TY, Ngan HY: Quantification of human papillomavirus DNA in the plasma of patients with cervical cancer. Int J Gynecol Cancer 2004, 14(5):903-910.

2. Robinson-Bennett BL, Deford J, Diaz-Arrastia C, Levine L, Wang HQ, Hannigan EV, Papaconstantinou J: Implications of tyrosine phosphoproteomics in cervical carcinogenesis. J Carcinog 2008, 7:2.

3. Parkin DM, Bray F, Ferlay J, Pisani P: Global cancer statistics, 2002. CA Cancer J Clin 2005, 55(2):74-108.

4. Nishio S, Katsumata N, Matsumoto K, Tanabe H, Yonemori K, Kohno T, Shimizu C, Ando M, Kasamatsu T, Fujiwara Y: Analysis of the clinicopathological prognosis of stage IVb cervical carcinoma. Oncol Rep 2008, 19(2):497-503.

5. Witczak CA, Sharoff CG, Goodyear L: AMP-activated protein kinase in skeletal muscle: from structure and localization to its role as a master regulator of cellular metabolism. Cell Mol Life Sci 2008, 65(23):3737-3755.

6. Carling D: AMP-activated protein kinase: balancing the scales. Biochimie 2005, 87(1):87-91.

7. Hardie DG: AMPK: a key regulator of energy balance in the single cell and the whole organism. Int J Obes (Lond) 2008, 32(Suppl 4):S7-S12.

8. Shackelford DB, Shaw RJ: The LKB1-AMPK pathway: metabolism and growth control in tumour suppression. Nat Rev Cancer 2009, 9(8):563-575.

9. Kuhajda FP: AMP-activated protein kinase and human cancer: cancer metabolism revisited. Int J Obes (Lond) 2008, 32(Suppl 4):S36-S41.

10. Yu SY, Chan DW, Liu WW, Ngan HY: Inhibition of cervical cancer cell growth through activation of upstream kinases of AMP-activated protein kinase. Tumour Biol 2009, 30(2):80-85.

11. Fay JR, Steele V, Crowell JA: Energy homeostasis and cancer prevention: the AMP-activated protein kinase. Cancer Prev Res (Phila) 2009, 2(4):301-309.

12. Kwan HT, Chan DW, Cai PC, Mak CS, Yung MM, Leung TH, Wong OG, Cheung AN, Ngan HY: AMPK activators suppress cervical cancer cell growth through inhibition of DVL3 mediated Wnt/beta-catenin signaling activity. PLoS One 2013, 8(1):e53597.

13. Wierstra I, Alves J: FOXM1, a typical proliferation-associated transcription factor. Biol Chem 2007, 388(12):1257-1274.

14. Wang X, Kiyokawa H, Dennewitz MB, Costa RH: The Forkhead Box m1b transcription factor is essential for hepatocyte DNA replication and mitosis during mouse liver regeneration. Proc Natl Acad Sci USA 2002, 99(26):16881-16886.

15. Katoh M: Human FOX gene family (Review). Int J Oncol 2004, 25(5):1495-1500. 
16. Kim IM, Ramakrishna S, Gusarova GA, Yoder HM, Costa RH, Kalinichenko W: The forkhead box $\mathrm{m} 1$ transcription factor is essential for embryonic development of pulmonary vasculature. J Biol Chem 2005, 280(23):22278-22286.

17. Yoshida Y, Wang IC, Yoder HM, Davidson NO, Costa RH: The forkhead box M1 transcription factor contributes to the development and growth of mouse colorectal cancer. Gastroenterology 2007, 132(4):1420-1431.

18. Chan DW, Yu SY, Chiu PM, Yao KM, Liu WW, Cheung AN, Ngan HY: Overexpression of FOXM1 transcription factor is associated with cervical cancer progression and pathogenesis. J Pathol 2008, 215(3):245-252.

19. Wilson MS, Brosens JJ, Schwenen HD, Lam EW: FOXO and FOXM1 in Cancer: The FOXO-FOXM1 Axis Shapes the Outcome of Cancer Chemotherapy. Curr Drug Targets 2011, 12(9):1256-1266.

20. Zhao F, Lam EW: Role of the forkhead transcription factor FOXO-FOXM1 axis in cancer and drug resistance. Front Med 2012, 6(4):376-380.

21. Leung TW, Lin SS, Tsang AC, Tong CS, Ching JC, Leung WY, Gimlich R, Wong GG, Yao KM: Over-expression of FoxM1 stimulates cyclin B1 expression. FEBS Lett 2001, 507(1):59-66.

22. Fung TK, Poon RY: A roller coaster ride with the mitotic cyclins. Semin Cell Dev Biol 2005, 16(3):335-342.

23. Wang IC, Chen YJ, Hughes D, Petrovic V, Major ML, Park HJ, Tan Y, Ackerson T, Costa $\mathrm{RH}$ : Forkhead box $\mathrm{M} 1$ regulates the transcriptional network of genes essential for mitotic progression and genes encoding the SCF (Skp2-Cks1) ubiquitin ligase. Mol Cell Biol 2005, 25(24):10875-10894.

24. Laoukili J, Kooistra MR, Bras A, Kauw J, Kerkhoven RM, Morrison A, Clevers H, Medema $\mathrm{RH}$ : FoxM1 is required for execution of the mitotic programme and chromosome stability. Nat Cell Biol 2005, 7(2):126-136.

25. Lok GT, Chan DW, Liu WW, Hui WW, Leung TH, Yao KM, Ngan HY: Aberrant activation of ERK/FOXM1 signaling cascade triggers the cell migration/ invasion in ovarian cancer cells. PLoS One 2011, 6(8):e23790.

26. Lam AK, Ngan AW, Leung MH, Kwok DC, Liu W, Chan DW, Leung WY, Yao KM: FOXM1b, which is present at elevated levels in cancer cells, has a greater transforming potential than FOXM1c. Front Oncol 2013, 3:11.

27. Chan DW, Hui WW, Cai PC, Liu MX, Yung MM, Mak CS, Leung TH, Chan KK, Ngan HY: Targeting GRB7/ERK/FOXM1 signaling pathway impairs aggressiveness of ovarian cancer cells. PLoS One 2012, 7(12):e52578.

28. Teh MT, Wong ST, Neill GW, Ghali LR, Philpott MP, Quinn AG: FOXM1 is a downstream target of Gli1 in basal cell carcinomas. Cancer Res 2002, 62(16):4773-4780

29. Madureira PA, Varshochi R, Constantinidou D, Francis RE, Coombes RC Yao KM, Lam EW: The Forkhead box M1 protein regulates the transcription of the estrogen receptor alpha in breast cancer cells. J Biol Chem 2006 281(35):25167-25176.

30. Kim IM, Ackerson T, Ramakrishna S, Tretiakova M, Wang IC, Kalin TV, Major ML, Gusarova GA, Yoder HM, Costa RH, et al: The Forkhead Box $\mathrm{m} 1$ transcription factor stimulates the proliferation of tumor cells during development of lung cancer. Cancer Res 2006, 66(4):2153-2161.

31. Douard $R$, Moutereau $S$, Pernet $P$, Chimingqi $M$, Allory $Y$, Manivet $P$, Conti $M$, Vaubourdolle M, Cugnenc PH, Loric S: Sonic Hedgehog-dependent proliferation in a series of patients with colorectal cancer. Surgery 2006, 139(5):665-670.

32. Kalinichenko W, Major ML, Wang X, Petrovic V, Kuechle J, Yoder HM, Dennewitz MB, Shin B, Datta A, Raychaudhuri $P$, et al: Foxm1b transcription factor is essential for development of hepatocellular carcinomas and is negatively regulated by the p19ARF tumor suppressor. Genes Dev 2004, 18(7):830-850.

33. Ahmad A, Wang Z, Kong D, Ali S, Li Y, Banerjee S, Ali R, Sarkar FH: FoxM1 down-regulation leads to inhibition of proliferation, migration and invasion of breast cancer cells through the modulation of extra-cellular matrix degrading factors. Breast Cancer Res Treat 2010, 122(2):337-346.

34. Kim YH, Liang H, Liu X, Lee JS, Cho JY, Cheong JH, Kim H, Li M, Downey TJ, Dyer MD, et al: AMPKalpha modulation in cancer progression: multilayer integrative analysis of the whole transcriptome in Asian gastric cancer. Cancer Res 2012, 72(10):2512-2521.

35. Guan TJ, Qin FJ, Du JH, Geng L, Zhang YY, Li M: AICAR inhibits proliferation and induced S-phase arrest, and promotes apoptosis in CaSki cells. Acta Pharmacol Sin 2007, 28(12):1984-1990.

36. Din FV, Valanciute A, Houde VP, Zibrova D, Green KA, Sakamoto K, Alessi DR, Dunlop MG: Aspirin inhibits mTOR signaling, activates AMP-activated protein kinase, and induces autophagy in colorectal cancer cells. Gastroenterology 2012, 142(7):1504-1515. e1503.
37. Karuman P, Gozani O, Odze RD, Zhou XC, Zhu H, Shaw R, Brien TP, Bozzuto CD, Ooi D, Cantley LC, et al: The Peutz-Jegher gene product LKB1 is a mediator of p53-dependent cell death. Mol Cell 2001, 7(6):1307-1319.

38. Liang J, Shao SH, Xu ZX, Hennessy B, Ding Z, Larrea M, Kondo S, Dumont DJ, Gutterman JU, Walker CL, et al: The energy sensing LKB1-AMPK pathway regulates p27(kip1) phosphorylation mediating the decision to enter autophagy or apoptosis. Nat Cell Biol 2007, 9(2):218-224.

39. Short JD, Houston KD, Dere R, Cai SL, Kim J, Johnson CL, Broaddus RR, Shen J, Miyamoto S, Tamanoi F, et al: AMP-activated protein kinase signaling results in cytoplasmic sequestration of p27. Cancer Res 2008, 68(16):6496-6506.

40. Tiainen M, Vaahtomeri K, Ylikorkala A, Makela TP: Growth arrest by the LKB1 tumor suppressor: induction of p21(WAF1/CIP1). Hum Mol Genet 2002, 11(13):1497-1504.

41. Zou Y, Tsai WB, Cheng CJ, Hsu C, Chung YM, Li PC, Lin SH, Hu MC: Forkhead box transcription factor FOXO3a suppresses estrogendependent breast cancer cell proliferation and tumorigenesis. Breast Cancer Res 2008, 10(1):R21.

42. Schmidt M, Fernandez de Mattos S, van der Horst A, Klompmaker R, Kops GJ, Lam EW, Burgering BM, Medema RH: Cell cycle inhibition by FoxO forkhead transcription factors involves downregulation of cyclin D. Mol Cell Biol 2002, 22(22):7842-7852.

43. Myatt SS, Lam EW: The emerging roles of forkhead box (Fox) proteins in cancer. Nat Rev Cancer 2007, 7(11):847-859.

44. Fernandez De Mattos S, Villalonga P, Clardy J, Lam EW: FOXO3a mediates the cytotoxic effects of cisplatin in colon cancer cells. Mol Cancer Ther 2008, 7(10):3237-3246.

45. Brunet A, Bonni A, Zigmond MJ, Lin MZ, Juo P, Hu LS, Anderson MJ, Arden KC, Blenis J, Greenberg ME: Akt promotes cell survival by phosphorylating and inhibiting a Forkhead transcription factor. Cell 1999, 96(6):857-868.

46. Motta MC, Divecha N, Lemieux M, Kamel C, Chen D, Gu W, Bultsma Y, McBurney M, Guarente L: Mammalian SIRT1 represses forkhead transcription factors. Cell 2004, 116(4):551-563.

47. Chiacchiera F, Simone C: The AMPK-FoxO3A axis as a target for cancer treatment. Cell Cycle 2010, 9(6):1091-1096.

48. Link W, Oyarzabal J, Serelde BG, Albarran MI, Rabal O, Cebria A, Alfonso P, Fominaya J, Renner O, Peregrina S, et al: Chemical interrogation of FOXO3a nuclear translocation identifies potent and selective inhibitors of phosphoinositide 3-kinases. J Biol Chem 2009, 284(41):28392-28400.

49. Rattan R, Giri S, Hartmann LC, Shridhar V: Metformin attenuates ovarian cancer cell growth in an AMP-kinase dispensable manner. J Cell Mol Med 2011, 15(1):166-178

50. Li C, Liu WW, Chan DW, Yao KM, Ngan HY: LY294002 and metformin cooperatively enhance the inhibition of growth and the induction of apoptosis of ovarian cancer cells. Int J Gynecol Cancer 2012, 22(1):15-22.

doi:10.1186/1471-2407-13-327

Cite this article as: Yung et al:: Activation of AMPK inhibits cervical cancer cell growth through AKT/FOXO3a/FOXM1 signaling cascade. BMC Cancer 2013 13:327.

\section{Submit your next manuscript to BioMed Central and take full advantage of:}

- Convenient online submission

- Thorough peer review

- No space constraints or color figure charges

- Immediate publication on acceptance

- Inclusion in PubMed, CAS, Scopus and Google Scholar

- Research which is freely available for redistribution 